\title{
Long noncoding RNAs in renal diseases
}

\author{
Minghui Liu' ${ }^{1}$ and Jie Ren $2,3,45^{*}$
}

\begin{abstract}
Long noncoding RNAs (IncRNAs) play critical roles in eukaryotic gene regulation and diseases, rather than being merely transcriptional "noise". Over the past decade, the study of IncRNAs has emerged as a burgeoning field of research and expanded our knowledge of their functions and underlining mechanisms in both normal and malignant cells. However, IncRNAs are still one of the least understood groups of transcripts. Here, we review the classifications and functions of IncRNAs and their roles in renal diseases. This review will provide insights into the roles of IncRNAs in pathogenesis, diagnosis and therapeutics of renal diseases and indications of IncRNAs as potential targets for the treatment of kidney diseases.
\end{abstract}

Keywords: IncRNAs, Renal diseases

\section{Introduction}

Eukaryotic genomes transcribe a broad spectrum of RNA molecules, with extensive diversity in their abundance, size and protein-coding capacities. Remarkably, only less than $2 \%$ of the human genome is transcribed into protein-coding RNA [1]. The rest of human genome is also mostly transcribed, but into a huge array of RNAs without the capability of coding proteins, hence the name "noncoding RNAs". They were initially considered as transcriptional noises or the dark matter of biology [2]. Till many years after, an increasing number of noncoding RNAs, especially miRNAs (microRNAs), piRNAs (Piwi-associated RNAs) and lncRNAs, were found to have crucial functions in gene regulation and are heavily involved in multiple physiological and pathological processes [1].

Unlike well-studied microRNAs, little is known about functions and underlining mechanisms of lncRNAs. In this important emerging field, researches over the past decade have shown that IncRNAs interfere with tissue homeostasis and play a role in renal pathological processes. However, to date, the study of lncRNAs in renal diseases is still in its infancy. In this review, we summarize available studies indicating that lncRNAs are heavily involved in kidney development and disease, and

\footnotetext{
* Correspondence: jren@cshl.edu

${ }^{2}$ Beijing Institute of Genomics, Chinese Academy of Sciences, Beijing, China ${ }^{3}$ Institute for Stem Cell and Regeneration, Chinese Academy of Sciences, Beijing, China

Full list of author information is available at the end of the article
}

propose lncRNAs as novel biomarkers for clinical diagnosis and potential therapeutic targets in renal diseases.

\section{Identification of IncRNAs}

LncRNAs, defined as noncoding RNA molecules longer than 200 nucleotides, were described with an emphasis initially in 2002 by Okazaki et al. in a large-scale sequencing study of full-length cDNA libraries in mice [3]. However, it is very difficult to distinguish lncRNAs from protein-coding transcripts. Although protein-coding transcripts are commonly characterized by the presence of an open reading frame (ORF) with more than 100 amino acids, some lncRNAs may also be predicted to contain such a long ORF [4]. Besides, some transcripts can be transformed between coding and non-coding isoforms. For example, SRA (steroid receptor RNA activator), a well-characterized IncRNA, can also encode a protein that functions antagonistically to its alternative roles as lncRNA [5]. On the other hand, p53 mRNA, the messenger RNA (mRNA) coding for a tumor suppressor, can also bind the Mdm2 (Mouse double minute 2 homolog) protein and function as a regulator directly at the RNA level [6]. To date, systematic methods for lncRNA identification have not been fully established, while a few commonly recognized criteria exists, such as sequence structure, size, presence of ORFs and codon substitution frequency.

(c) The Author(s). 2019 Open Access This article is distributed under the terms of the Creative Commons Attribution 4.0 International License (http://creativecommons.org/licenses/by/4.0/), which permits unrestricted use, distribution, and reproduction in any medium, provided you give appropriate credit to the original author(s) and the source, provide a link to the Creative Commons license, and indicate if changes were made. The Creative Commons Public Domain Dedication waiver (http://creativecommons.org/publicdomain/zero/1.0/) applies to the data made available in this article, unless otherwise stated. 


\section{Classifications of IncRNAs}

As a broad concept, lncRNAs encompass a few types of RNA transcripts. According to their location in the genome, lncRNAs can be classified into seven broad categories as following: (a) sense lncRNAs, (b) antisense lncRNAs, (c) bidirectional lncRNAs, (d) intronic lncRNAs, (e) intergenic lncRNAs and (f) enhancer lncRNAs (illustrated in Fig. 1). When it comes to concrete functions, lncRNAs can be divided into four groups, namely, (a) signal lncRNAs, (b) decoy lncRNAs, (c) guide lncRNAs and (d) scaffold lncRNAs (described in Fig. 2). LncRNAs can be found within the nuclear or cytoplasmic fractions. Cytoplasmic lncRNAs can work as microRNA sponges or miRNA precursors to either reduce or increase the expression and function of microRNAs [4]. They can also recognize target mRNAs to interact with the cellular translational machinery [4]. Nuclear lncRNAs exert its effects on chromatin architecture either in a cis-acting way (lncRNAs regulate the expression of neighboring genes) or in a trans-acting way (lncRNAs regulate the expression of distant genes).
Besides, for some nuclear lncRNAs, it is not clear whether they function in cis or trans [7].

\section{Functions of IncRNAs}

LncRNAs can regulate gene expression to affect many important physiological processes in multiple roles, to name only a few, as chromatin modifiers, $\mathrm{X}$ chromosome inactivator, enhancers, transcriptional regulators and post-transcriptional regulators.

\section{Chromatin modifiers}

LncRNAs have been demonstrated to participate in chromatin modification in a critical way, which subsequently affects multiple important biological processes including neurogenesis and stem cell pluripotency [8]. LncRNAs regulate the state of chromatin by recruiting chromatin remodeling proteins to specific genomic loci. For instance, Hox genes are a class of homeotic genes related to the temporal and spatial developmental axes where hundreds of lncRNAs have been shown to be critical [9]. One of these lncRNAs, HOTAIR (Hox

(a) sense IncRNAs

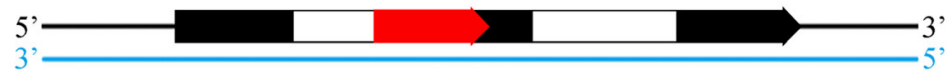

(b) antisense IncRNAs

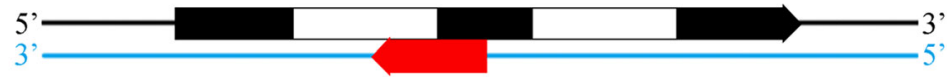

(c) bidirectional lncRNAs

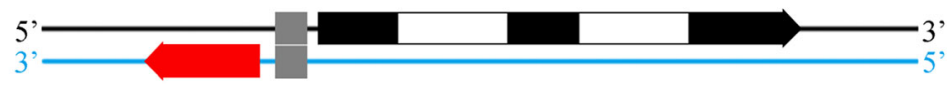

(d) intronic lncRNAs

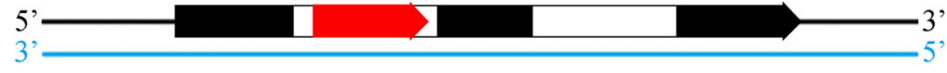

(e) intergenic lncRNAs

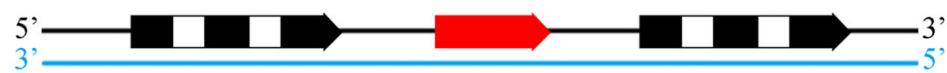

(f) enhancer $\operatorname{lncRNAs}$

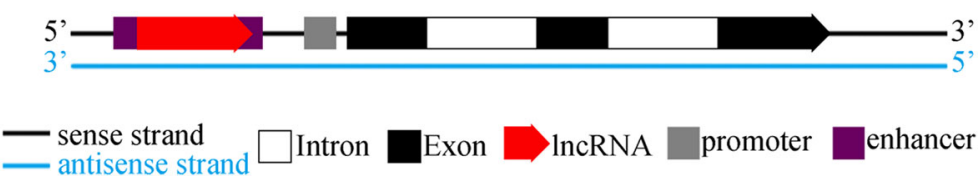

Fig. 1 Illustration of the classifications of IncRNAs. a Sense IncRNAs are transcribed from the same strand of protein-coding genes, overlapping exons from the protein-coding genes. $\mathbf{b}$ Antisense IncRNAs are transcribed from the opposite strand of protein-coding genes, overlapping exons of protein-coding genes on the antisense strand. c Bidirectional IncRNAs are located on the opposite stand of protein-coding genes and transcribed from close proximity (less than 1000 base pairs) of their neighboring genes. $\mathbf{d}$ Intronic IncRNAs are derived entirely from introns of protein-coding genes. e Intergenic IncRNAs lie within intergenic regions. $\mathbf{f}$ Enhancer IncRNAs originate from the enhancer regions of protein-coding genes 
(a) signal lncRNAs

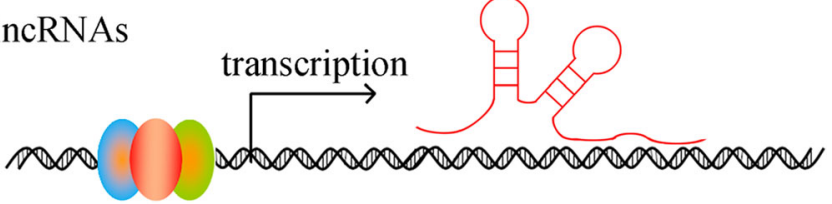

(b) decoy IncRNAs

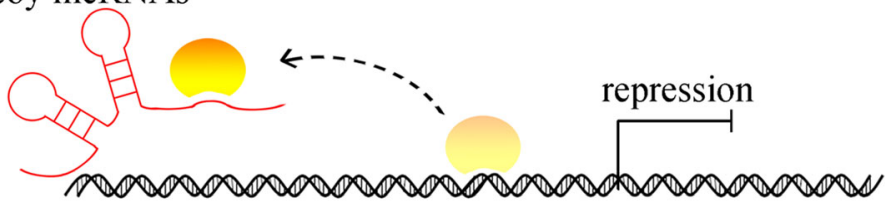

(c) guide lncRNAs

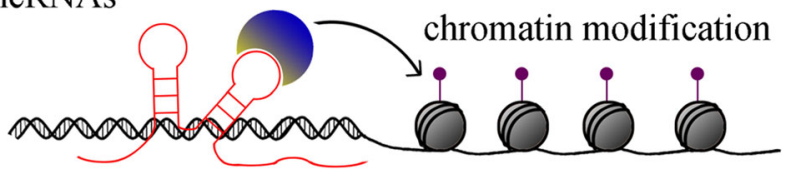

(d) scaffold lncRNAs

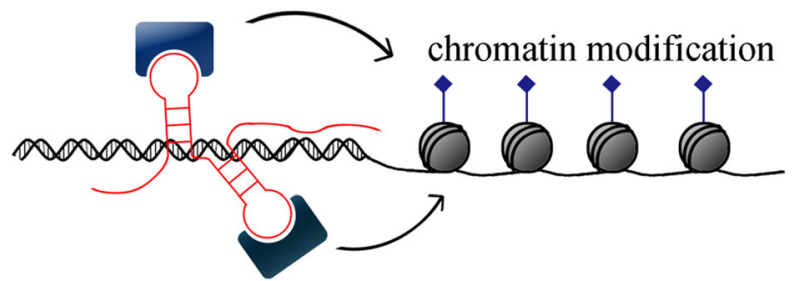

Fig. 2 Schematic diagram of the modes of action of IncRNAs. a Signal IncRNAs are transcribed with spatiotemporal precision and expressed in response to developmental cues, concerting the combinatorial actions of transcription factors (shown as colored ovals) to regulate gene expressions. $\mathbf{b}$ Decoy IncRNAs act as competing endogenous RNA molecules that titrate away transcription factors or other proteins from the chromatin to exert functions like repressing the transcription of target mRNAs. c Guide IncRNAs recruit ribonucleoprotein complexes to target genes for chromatin modification. $\mathbf{d}$ Scaffold IncRNAs bring multiple enzymes together to form a chromatin-modifying complex that either suppresses or initiates transcription

transcript antisense RNA) originates from HoxC locus and silences HoxD genes spanning over $40 \mathrm{~kb}$ by recruiting PRC2 (Polycomb repressive complex-2) in a trans-acting way, finally leading to a repressive chromatin state [9]. To be noted, PRC2 is a histone methyltransferase required for epigenetic silencing and thereby an important chromatin modifying factor. Besides HOTAIR, thousands of RNAs can bind PRC2 in vivo, although this raises questions about binding specificity and function in different chromatin contexts [10]. Nevertheless, it has been a prototype for lncRNAs interacting with PRC2 to alter the chromatin state [11]. Other well-studied lncRNAs known to bind PRC2 include Xist (X-inactive specific transcript) [12], Kcnq1ot1 (KCNQ1 overlapping transcript 1) [13], Braveheart [14], ANRASSF1 [15], etc. For example, Kcnq1ot1 is a lncRNA acting as an important mediator for imprinting. The promoter of Kcnq1ot1 maps to the ICRs (imprinting control regions) of Kcnq1 gene, which encodes a protein for a voltage-gated potassium channel responsible for the repolarization of the cardiac action potential. Kcnq1ot1 interacts with Dnmt1 (DNA (cytosine-5)-methyltransferase 1) to establish the placental-specific imprinting of genes within the Kcnq1 domain [16]. Besides, Kcnq1ot1 induces the methylation of histone H3 on lysine 9 and lysine 27 by recruiting the histone methyltransferases G9a and PRC2 [13]. Xist is required for the $\mathrm{X}$-inactivation process during early development in female mammals, as only one $\mathrm{X}$ chromosome will remain active. The other X chromosomes expressing Xist will be coated with it and packaged into a transcriptionally-inactive heterochromatic structure [12]. In this process, Xist will recruit a series of proteins including PRC2, SPEN, SAF-A (Scaffold Attachment Factor-A) and LBR to initiate the $\mathrm{X}$ chromosome inactivation in cis [17], while leaving the other $\mathrm{X}$ chromosome lacking Xist active [12]. Another lncRNAs, Firre is also involved in the $\mathrm{X}$-inactivation process by anchoring the inactive $\mathrm{X}$ 
chromosome to the position adjacent to the nucleolus [17]. To sum up, nuclear lncRNAs exerts its effect on chromatin state mostly through interaction with chromatin modifying proteins.

\section{Enhancers}

A subset of lncRNAs are transcribed from active enhancers and promote the expression of corresponding protein-coding genes in return, hence the name enhancer IncRNAs. In 2010, Kim et al. coined the concept of enhancer RNAs based on the phenomena that RNA polymerase II located to approximately 3000 activated enhancers and that RNAs could be produced from the extragenic enhancer regions of protein-coding genes [18]. Almost at the same time, Shiekhattar lab reported lncRNAs with enhancer-like functions. They characterized several cis-acting lncRNAs using GENCODE annotation of the human genome and discovered an RNA-dependent potentiation of gene expression mediated by the ncRNA-a1-7 in particular [19]. Besides, the Evf2 noncoding RNA, which originates from the Dlx-5/6 ultraconserved region, can interact with Dlx-2 to induce transcriptional enhancement of Dlx-2 in a target and homeodomain-specific way [20]. The HSR1 (heat shock RNA-1), which is constitutively expressed in human and rodent cells, works along with eEF1A and actively mediates the activation process of the HSF1 (heat-shock transcription factor 1) [21]. Steroid receptor RNA activator (SRA) also acts as a noncoding transcript to coactivate steroid receptor [5]. Rosenfeld et al. also reported that PRNCR1 and PCGEM1 bind to the AR (androgen receptor) and potently enhance the AR-mediated gene activation programs in prostate cancer cells [22]. However, so far, the functional mechanism of enhancer lncRNAs have not yet been firmly established. More efforts need to be made into revealing the secrets of enhancer lncRNAs in various biological processes.

\section{Transcriptional regulators}

Transcriptional regulations of eukaryotic genes are achieved through many ways, including traditional direct interactions of proteins with DNA regulatory elements and, more recently identified, specific interactions between RNAs, DNAs and/or proteins. Thus, lncRNAs are now known as an important facet of such transcriptional regulations. For cis-acting lncRNA, its genomic origin is critical for its function, as it will alter the expression of protein-coding genes nearby. It may function through the transcription activity itself rather than the product: if the promoter of another gene lies in close proximity, it may cause the collision between transcriptional machineries on both genes, which is also dubbed "transcriptional interference". For example, active transcription of lncRNA SRG1 will repress the transcription of its downstream SER3 gene in yeast, because the 3' end of SRG1 overlap with the SER3 promoter. If SRG1 transcription is prematurely terminated, the repression of SER3 will be alleviated [2]. Also, in yeast, transcription of some lncRNAs facilitates the accessibility of protein-coding genes to RNA polymerases through altering chromatin structure, such as promoting transcription initiation at the FBP1 (Fructose-1,6-bisphosphatase 1) gene [23]. On the other hand, lncRNAs may also act in trans, affecting transcription via its binding to transcription factors. For instance, the lncRNA 7SK binds to the elongation factor $\mathrm{P}-\mathrm{TEFb}$ and downregulates its kinase activity in order to inhibit transcription elongation by Pol II [24].

\section{Post-transcriptional regulators}

LncRNAs exert post-transcriptional regulation mainly in two ways, splicing regulation and translational control. Firstly, lncRNAs can either bind to splicing factors in a competition manner or bind to mRNA itself through base-pairing to block mRNA splicing. MALAT-1 (Metastasis-associated in lung adenocarcinoma transcript-1) is an abundant $\sim 7 \mathrm{~kb}$ lncRNA that interacts with the serine/arginine-rich (SR) splicing factors. It was suggested to regulate phosphorylation of SR proteins to modulate their distribution in nuclear speckles, thereby affecting alternative splicing of pre-mRNAs [25]. MIAT (Myocardial infarction associated transcript) is another IncRNA containing a highly-conserved tandem repeats of UACUAAC, which has been characterized as a conserved intron branch point that can bind SF1 (splicing factor 1) with a higher affinity than the divergent branch point sequence, thus repressing splicing and the formation of spliceosomal complex on other transcripts [26]. LUST (LUCA-15-specific transcript) is an antisense transcript of RBM5 (RNA binding motif protein 5) and a sense-strand regulatory sequence of RBM5 in disguise, which is considered to modulate the expression of RBM5 splicing variants [27]. Secondly, lncRNAs can bind ribosome or translation factors to control protein translation. For example, snaR (small NF90-associated RNAs) and Gadd7 (growth arrested DNA-damage inducible gene 7) are two examples of translational control by lncRNAs through binding to ribosomes [28]. On the other hand, BC1 (Brain cytoplasmic RNA 1) and BC200 (200 nt brain cytoplasmic RNA), represent examples of translational repression by lncRNAs through binding to translation factors such as eI4FA (eukaryotic translation initiation factor 4A), PABP (poly (A)-binding protein) and other factors [29]. Thirdly, some lncRNAs can be related to both splicing and translation. One example is Zeb2NAT (Zeb2 natural antisense transcript), a $\sim 1.2 \mathrm{~kb}$ lncRNA overlapping the $5^{\prime}$ splicing site of an intron, whose retention is required for the translation of Zeb2 
(zinc finger E-box binding homeobox 2). Zeb2NAT can repress the intron splicing and thereby promote Zeb2 translation [30]. Finally, lncRNAs can also function as microRNA sponges or microRNA precursors to participate in the post-transcriptional modulation. MicroRNAs are a class of small single-stranded RNAs without protein-coding capacity. MicroRNAs can repress mRNA translation or facilitate mRNA degradation via binding to the 3'-UTR (3' untranslated region) of their target mRNAs. In that case, a few IncRNAs can alter mRNA levels through influencing corresponding microRNA levels. H19, a well-studied IncRNA, functions as a molecular sponge to sequester let-7, while at the same time serves as a precursor of miR-675-3p [31, 32]. LncRNAs with similar functions have been reported and summarized in reference [33].

\section{Research methods for IncRNAs}

Generally, similar experimental procedures have been used for quantification and identification of IncRNA as coding transcripts although with some modification in downstream processing. LncRNA are often quantified together with mRNA using sequencing or microarray techniques, RNA-seq and -chip respectively, in the same biological samples. RNA-seq has the advantage to identify novel RNA transcripts and developed rapidly in the last decades. In addition to the common application of next-generation sequencing (NGS), recent advances in RNA-seq include single cell sequencing [34], single molecule sequencing [35], and in situ sequencing of fixed tissue [36]. On the other hand, transcriptome microarray is still in use and provides benefits as equally well-developed data analyses with a lower stochastic variability. Particularly in clinical studies, microarrays even outperform RNA-seq for standard analysis of gene expressions when it comes to reproducibility and cost [37].

In terms of functional analysis for lncRNAs, small interfering RNAs or antisense oligonucleotides for knocking down targeted lncRNA, and overexpression constructs to increase certain lncRNA expression levels are traditional methods to reveal their roles in vivo. In recent years, the revolutionizing CRISPR (clustered regularly interspaced short palindromic repeats) systems have been incorporated to maneuver the transcript level by either CRISPR activation or CRISPR inhibin (CRIS$\mathrm{PRa} / \mathrm{i})[38,39]$ or for genome editing of lncRNA locus of interest. For nuclear lncRNAs, to study the associations between IncRNAs and chromatin, ChIRP (Chromatin Isolation by RNA Purification) [40], CHART (capture hybridization analysis of RNA targets) [41], RAP (RNA antisense purification) [42] and GRID-seq (capture in situ global RNA interactions with DNA by deep sequencing) [43] are among the most advanced technologies to identify the binding sites of IncRNAs genome-wide.
ChIRP, CHART and RAP can study only one known IncRNA, while GRID-seq provides global detection and analysis of RNA-chromatin interactions with high specificity and sensitivity [43]. To explore the interactions between IncRNAs and proteins, RIP (RNA immunoprecipitation) [44] and CLIP (UV crosslinking and immunoprecipitation) [45], iCLIP (individual-nucleotide resolution CLIP) [46] can be utilized to capture the lncRNA-binding proteins. Similar strategies can be applied to cytoplasmic lncRNAs, which usually function as miRNA sponges or precursors. In addition, with accumulating studies of lncRNA, a number of databases have emerged over the past few years with a particular interest in curating lncRNAs, to name only a few, NONCODE [47], ChipBase [48], IncRNAdb [49], LNCipedia [50] and LncRNADisease [51].

\section{LncRNAs in renal diseases}

There is an increasing number of evidences showing the important roles of lncRNAs in diverse human diseases. However, studies on lncRNAs in renal diseases are still in its infancy and mainly restricted to renal cancer. There are very few reports on lncRNAs' function in other types of renal diseases, such as acute kidney injury, renal fibrosis, polycystic kidney disease, diabetic kidney disease, lupus nephritis and renal transplantation. Here, we discuss major researches published so far on lncRNAs in renal diseases.

\section{Renal cancer}

As lncRNAs play important roles in regulating major pathways in cell growth, proliferation, differentiation, apoptosis and survival, dysregulation of lncRNAs can promote tumorigenesis and progression of kidney cancer, especially in renal cell carcinoma (RCC). For example, the oncogenic IncRNA HOTAIR promotes RCC tumorigenesis through AXL signaling by acting as a ceRNA (competing endogenous RNA) to sequester miR-217, a tumor suppressor, to facilitate HIF-1 (hypoxia-inducible factor 1) expression and to upregulate AXL level. The lncRNA-MRCCAT1 (metastatic renal cell carcinoma-associated transcript 1 ) is highly expressed in clear cell renal cell carcinoma (ccRCC) and promotes metastatic properties of ccRCC [52]. Besides, the upregulations of IncRNA-UCA1 (urothelial carcinoma associated 1) [53], IncRNA-ATB [54], lncRNA-H19 [55] and lncRNA-FTX [56] are also involved in RCC tumorigenesis and proposed to be important biomarkers for RCC. On the other hand, lncRNAs can also play anti-tumor roles. For example, lncRNA-SARCC (Suppressing Androgen Receptor in Renal Cell Carcinoma) is differentially modulated in a VHL (von Hippel-Lindau)-dependent way under hypoxia, suppressing the proliferation of 
VHL-mutant RCC cell yet promoting the growth of VHL-normal RCC cell [57]. Decreases of lncRNAs such as NBAT-1 (neuroblastoma associated transcript-1) [58] and CASC2 [59] (cancer susceptibility candidate 2) are associated with poor prognosis in patients with RCC. To date, novel lncRNAs continue to be identified, such as lnc-BMP2-2, lnc-CPN2-1, lnc-ACACA-1, lnc-FOXG1-2 and lnc-TTC34-3, which were predicted by computational analyses to participate in RNA-protein interaction networks including spliceosome and other complexes in RCC [60]. Remarkably, it is reported that intronic antisense lncRNAs are commonly expressed in RCC tumors, the majority of which is evolutionarily conserved and possibly modulated by epigenetic modifications [61]. Besides, a recent study shows that lncRNA can also be packaged into exosomes and function critically to promote the progress of renal carcinoma. Le et al. identified lncARSR (lncRNA activated in RCC with sunitinib resistance), which acts as competing endogenous RNA for miR-34 and miR-449 to promote c-MET and AXL expression. In addition, lncARSR can be packaged into exosomes and transmitted to sensitive cells to disseminate sunitinib resistance [62]. Although studies are accumulating over the past decade, our knowledge of the underlying mechanisms of lncRNAs' role in renal malignancies is still rudimental, which needs more efforts to be made.

\section{Acute kidney injury}

Studies on lncRNAs in acute kidney injury (AKI) can be counted on fingers. One case is the hypoxia-responsive lncRNA GAS5, which is upregulated in renal ischemia/ reperfusion (I/R) injury along with the increased expressions of p53 and TSP-1 (thrombospondin 1), promoting cell apoptosis in kidney [63]. Another case is the lncRNA MALAT-1, which is highly expressed in ischemic kidneys and plasma samples of patients with AKI, indicating a potential role of MALAT-1 in the induction of AKI [64]. However, MALAT-1 was later reported to be dispensable for renal I/R injury [65]. LncRNA-PRINS (psoriasis susceptibility-related RNA gene induced by stress) is induced by HIF- $1 \alpha$ under hypoxia and shows specific interaction with RANTES (regulated on activation, normal T-cell expressed and secreted protein), which recruits circulating leukocytes and aggravates kidney injury [66]. In addition, Lorenzen et al. identified a novel intronic antisense lncRNA as an independent predictor of mortality in critically Ill patients with AKI, dubbed TapSAKI (transcript predicting survival in AKI), which is enriched in tubular epithelial cells and increased in plasma samples of AKI patients [67]. However, it still remains elusive whether these circulating
lncRNAs are incorporated into exosomes and secreted into the circulating system.

\section{Renal fibrosis / chronic kidney disease}

The lncRNA np_5318 is a novel intronic lncRNA, which is located between the first and second exons of Erbb4 in mouse genome and thereby named as Erbb4-IR. Feng et al. reported that TGF-beta 1 can highly increase Erbb4-IR expression via a Smad3-dependent manner in the fibrotic kidney of mouse, suggesting that Erbb4-IR is a specific therapeutic target for chronic kidney disease [68]. Wang et al. reported the lncRNA ZEB1-AS1 (zinc finger E-box binding homeobox1-antisense RNA 1) exerted an anti-fibrotic role in diabetic nephropathy [69]. Xie et al. reported IncRNA H19 overexpression promoted renal fibrosis [70]. Chen et al. characterized downregulation of an intergenic lncRNA LINC00963, which suppresses RIF (renal interstitial fibrosis) and OS (oxidative stress) of CRF (chronic renal failure) through activation of the FoxO (forkhead box $\mathrm{O}$ ) signaling pathway [71]. Zhou et al. demonstrated that lncRNA HOTAIR participated in renal interstitial fibrosis through upregulating miR-124 to block Notch1 pathway [72].

\section{Diabetic nephropathy}

Hundreds of lncRNAs are deregulated in diabetic nephropathy (DN) [73], which might participate in pathogenesis of $\mathrm{DN}$, according to a recent study using microarray analysis on lncRNAs in DN. However, only a handful of these deregulated IncRNAs have been demonstrated to affect proliferation and fibrosis in DN, including CYP4B1-PS1-001 [74], ENSMUST00000147869 [75], NR_033515 [76], Dlx6os1 [77] and LINC00968 [78]. Yet, several lncRNAs can be potential therapeutic targets for DN, such as MALAT1. Reducing MALAT1 levels can improve renal functions after duodenal-jejunal bypass in diabetic rats [79]. Increasing expression of lncRNA TUG1 (taurine upregulated gene 1) can attenuate podocyte apoptosis, alleviate extracellular matrix accumulation and protect diabetic rats from $\mathrm{DN}[80,81]$. Besides, TUG1 was also reported to regulate mitochondrial bioenergetics in DN [82].

\section{Polycystic kidney disease / lupus nephritis / glomerulonephritis}

There are few reports on lncRNAs in polycystic kidney disease, lupus nephritis or glomerulonephritis. One microarray study characterized thousands of lncRNAs in patients with IgA-negative MsPGN (mesangial proliferative glomerulonephritis) [83]. LncRNA Hoxb3os, which regulates mTOR signaling, was found to be deregulated in polycystic kidney disease [84]. The lncRNA NEAT1 (Nuclear Enriched Abundant Transcript 1) was 
characterized as a novel inflammatory mediator in human lupus. Yet it is still unclear whether renal functions in patients with lupus would be affected by NEAT1 [85].

\section{Kidney transplantation}

Even less studies have been carried out when it comes to lncRNAs in kidney transplantation. Chen et al. compared differential expressions of lncRNAs by microarray between control samples and samples from patients with acute rejection (AR) after renal transplantation, implying that lncRNAs might take part in the pathogenesis of AR [86]. Lorenzen et al. detected lncRNAs in urine of patients with $\mathrm{AR}$ and identified urinary IncRNA RP11-354P17.15-001 as a novel noninvasive biomarker for renal AR [87]. Ge et al. identified two lncRNAs (AF264622 and AB209021) from peripheral blood, which can predict renal AR following transplantation [88]. Shang et al. screened lncRNA expression patterns in recipients with urothelial cancer after kidney transplantation and suggested that lncRNAs have critical roles in UC carcinogenesis [89]. However, potential regulatory mechanisms of lncRNAs in renal transplantation are seldomly explored. Only two studies on lncRNA-ATB [90] and LncRNA-PRINS [91] did some initial research on the underlying mechanisms involved in the rejection of renal allografts.

\section{Future perspectives for the clinical use of IncRNAs in renal diseases}

As a newly developed research field, lncRNAs rapidly become a "hotspot" and provide new insights for potential clinical uses. On one hand, numerous lncRNAs have been discovered exhibiting specific expression patterns in various diseases, not only in tissues but also in body fluids including blood and urine. Thereby, lncRNAs could be developed for novel biomarkers to predict and supervise the progression of diseases. However, the quantity and stability of IncRNAs in the circulating and urinary systems make it hardly practical to use lncRNAs as non-invasive biomarkers with current detection limits. On the other hand, many lncRNAs have been demonstrated to directly regulate effector genes critical for the occurrence and development of diseases. From this standpoint, lncRNAs are also attractive therapeutic targets. For example, utilizing antisense oligonucleotides (ASOs) to knockdown MALAT1 can result in slower tumor growth and a reduction in metastasis in mammary tumors, indicating MALAT1 ASOs might provide a potential treatment for breast cancer [92]. In renal diseases, the potential clinical application of lncRNA studies are similar to other human diseases, focusing on biomarkers and therapeutic targets, and may provide new insights into diagnosis and therapy of renal diseases.
Nevertheless, up to now, no clinical trials of lncRNAs have been documented.

\section{Conclusion}

We summarized lncRNAs and their research status in renal diseases in this review. Although lncRNAs studies have increased a lot over the past decade, they are still at the starting stage regarding to kidney diseases for the time being. More efforts need to be made to explore the potential mechanisms and significant roles of lncRNAs during the pathogenesis, diagnosis and treatment of renal diseases, which will expand our understanding of renal disease pathophysiology and drive new strategies for the diagnosis and treatment for renal diseases.

\section{Abbreviations}

3'-UTR: 3' untranslated region; AKI: Acute kidney injury; AR: Acute rejection; AR: Androgen receptor; BC1: Brain cytoplasmic RNA 1; BC200: $200 \mathrm{nt}$ brain cytoplasmic RNA; CASC2: Cancer susceptibility candidate 2; cCRCC: Clear cell renal cell carcinoma; ceRNA: Competing endogenous RNA; DN: Diabetic nephropathy; Dnmt1: DNA (cytosine-5)-methyltransferase 1; el4FA: Eukaryotic translation initiation factor 4A; FBP1: Fructose-1,6-bisphosphatase 1;

FoxO: Forkhead box O; Gadd7: Growth arrested DNA-damage inducible gene 7; HIF-1: Hypoxia-inducible factor 1; HOTAIR: Hox transcript antisense RNA; HSF1: Heat-shock transcription factor 1; HSR1: Heat shock RNA-1; I/

R: Ischemia/reperfusion; ICRs: Imprinting control regions; Kcnq1 ot1: KCNQ1 overlapping transcript 1; LncRNA: Long noncoding RNA; LUST: LUCA-15specific transcript; MALAT-1: Metastasis-associated in lung adenocarcinoma transcript-1; Mdm2: Mouse double minute 2 homolog; MIAT: Myocardial infarction associated transcript; miRNAs: microRNAs; MRCCAT1: Metastatic renal cell carcinoma-associated transcript 1; mRNA: Messenger RNA; MsPGN: Mesangial proliferative glomerulonephritis; NBAT-1: Neuroblastoma associated transcript-1; NEAT1: Nuclear Enriched Abundant Transcript 1; ORF: Open reading frame; OS: Oxidative stress; PABP: Poly (A)-binding protein; piRNAs: piwi-associated RNAs; PRC2: Polycomb repressive complex-2; PRINS: Psoriasis susceptibility-related RNA gene induced by stress; RANTES: Regulated on activation, normal T-cell expressed and secreted; RBM5: RNA binding motif protein 5; RCC: Renal cell carcinoma; RIF: Renal interstitial fibrosis; SAF-A: Scaffold Attachment Factor-A; SARCC: Suppressing Androgen Receptor in Renal Cell Carcinoma; SF1: Splicing factor 1; snaR: Small NF90-associated RNAs; SRA: Steroid receptor RNA activator; TapSAKI: Transcript predicting survival in AKI; TSP-1: Thrombospondin 1; TUG1: Taurine upregulated gene 1; UCA1: Urothelial carcinoma associated 1; VHL: Von Hippel-Lindau; Xist: X-inactive specific transcript; ZEB1-AS1: Zinc finger E-box binding homeobox1-antisense RNA 1; Zeb2: Zinc finger E-box binding homeobox 2; Zeb2NAT: Zeb2 natural antisense transcript

\section{Acknowledgements}

Not applicable.

Funding

Supported by the Strategic Priority Research Program of the Chinese Academy of Sciences (XDA16010210).

Availability of data and materials Not applicable.

\section{Authors' contributions}

ML designed and drafted the manuscript. JR contributed to revising the manuscript. Both authors read and approved the final manuscript.

Ethics approval and consent to participate Not applicable.

Consent for publication

Not applicable. 


\section{Competing interests}

The authors declare that they have no competing interests.

\section{Publisher's Note}

Springer Nature remains neutral with regard to jurisdictional claims in published maps and institutional affiliations.

\section{Author details}

'State Key Laboratory of Pharmaceutical Biotechnology, NJU Advanced Institute for Life Sciences, Jiangsu Engineering Research Center for MicroRNA Biology and Biotechnology, Nanjing University, Nanjing, China. ${ }^{2}$ Beijing Institute of Genomics, Chinese Academy of Sciences, Beijing, China. Institute for Stem Cell and Regeneration, Chinese Academy of Sciences, Beijing, China. ${ }^{4}$ University of Chinese Academy of Sciences, Beijing, China. ${ }^{5}$ Cold Spring Harbor Laboratory, Cold Spring Harbor, NY, USA.

\section{Received: 15 October 2018 Accepted: 15 March 2019}

Published online: 02 December 2019

\section{References}

1. Ezkurdia I, et al. Multiple evidence strands suggest that there may be as few as 19000 human protein-coding genes. Hum Mol Genet. 2014 23(22):5866-78.

2. Ponting $C P$, Oliver $P L$, Reik W. Evolution and functions of Long noncoding RNAs. Cell. 2009:136(4):629-41.

3. Okazaki $Y$, et al. Analysis of the mouse transcriptome based on functional annotation of 60,770 full-length cDNAs. Nature. 2002; 420(6915):563-73.

4. Mercer TR, Dinger ME, Mattick JS. Long non-coding RNAs: insights into functions. Nat Rev Genet. 2009:10(3):155-9.

5. Chooniedass-Kothari $\mathrm{S}$, et al. The steroid receptor RNA activator is the first functional RNA encoding a protein. FEBS Lett. 2004:566(1-3):43-7.

6. Candeias MM, et al. p53 mrNA controls p53 activity by managing Mdm2 functions. Nat Cell Biol. 2008;10(9):1098-105.

7. Ma L, Bajic VB, Zhang Z. On the classification of long non-coding RNAs. RNA Biol. 2013;10(6):925-34

8. Ng S, Johnson R, Stanton LW. Human long non-coding RNAs promote pluripotency and neuronal differentiation by association with chromatin modifiers and transcription factors. EMBO J. 2012;31(3):522-33.

9. Rinn $J$, et al. Functional demarcation of active and silent chromatin domains in human HOX loci by noncoding RNAs. Cell. 2007;129(7):1311-23.

10. Davidovich $C$, et al. Toward a consensus on the binding specificity and promiscuity of PRC2 for RNA. Mol Cell. 2015:57(3):552-8.

11. Davidovich $C$, Cech TR. The recruitment of chromatin modifiers by long noncoding RNAs: lessons from PRC2. RNA. 2015;21(12):2007-22.

12. Zhao J, et al. Polycomb proteins targeted by a short repeat RNA to the mouse X chromosome. Science. 2008:322(5902):750-6.

13. Pandey RR, et al. Kcnq1 ot1 antisense noncoding RNA mediates lineagespecific transcriptional silencing through chromatin-level regulation. Mol Cell. 2008;32(2):232-46.

14. Klattenhoff CA, et al. Braveheart, a Long noncoding RNA required for cardiovascular lineage commitment. Cell. 2013;152(3):570-83.

15. Beckedorff FC, et al. The Intronic Long noncoding RNA ANRASSF1 recruits PRC2 to the RASSF1A promoter, reducing the expression of RASSF1A and increasing cell proliferation. PLoS Genet. 2013;9:e10037058.

16. Mohammad F, et al. Kcnq1ot1 noncoding RNA mediates transcriptional gene silencing by interacting with Dnmt1. Development. 2010;137(15): 2493-9.

17. Yang $F$, et al. The IncRNA firre anchors the inactive $X$ chromosome to the nucleolus by binding CTCF and maintains H3K27me3 methylation. Genome Biol. 2015;16:52.

18. Kim T, et al. Widespread transcription at neuronal activity-regulated enhancers. Nature. 2010:465(7295):182-U65.

19. Orom UA, et al. Long noncoding RNAs with enhancer-like function in human cells. Cell. 2010;143(1):46-58.

20. Feng J, et al. The Evf-2 noncoding RNA is transcribed from the Dlx-5/6 ultraconserved region and functions as a Dlx-2 transcriptional coactivator. Genes Dev. 2006;20(11):1470-84.

21. Shamovsky I, et al. RNA-mediated response to heat shock in mammalian cells. Nature. 2006;440(7083):556-60.
22. Yang $L$, et al. IncRNA-dependent mechanisms of androgen-receptorregulated gene activation programs. Nature. 2013;500(7464):598.

23. Hirota $K$, et al. Stepwise chromatin remodelling by a cascade of transcription initiation of non-coding RNAs. Nature. 2008:456(7218):130-U15.

24. Yik J, et al. Inhibition of P-TEFb (CDK9/Cyclin T) kinase and RNA polymerase II transcription by the coordinated actions of HEXIM1 and 7SK snRNA. Mol Cell. 2003;12(4):971-82.

25. Tripathi $V$, et al. The nuclear-retained noncoding RNA MALAT1 regulates alternative splicing by modulating SR splicing factor phosphorylation. Mol Cell. 2010;39(6):925-38.

26. Tsuiji $\mathrm{H}$, et al. Competition between a noncoding exon and introns: Gomafu contains tandem UACUAAC repeats and associates with splicing factor-1. Genes Cells. 2011;16(5):479-90.

27. Rintala-Maki ND, Sutherland LC. Identification and characterisation of a novel antisense non-coding RNA from the RBM5 gene locus. Gene. 2009; 445(1-2):7-16

28. Parrott AM, et al. The evolution and expression of the snaR family of small non-coding RNAs. Nucleic Acids Res. 2011;39(4):1485-500.

29. Lin D, et al. Translational control by a small RNA: dendritic BC1 RNA targets the eukaryotic initiation factor 4A helicase mechanism. Mol Cell Biol. 2008; 28(9):3008-19.

30. Beltran $M$, et al. A natural antisense transcript regulates Zeb2/Sip1 gene expression during Snail1-induced epithelial-mesenchymal transition. Genes Dev. 2008;22(6):756-69.

31. Kallen AN, et al. The imprinted H19 LncRNA antagonizes Let-7 MicroRNAs. Mol Cell. 2013:52(1):101-12.

32. Keniry $\mathrm{A}$, et al. The $\mathrm{H} 19$ lincRNA is a developmental reservoir of miR-675 that suppresses growth and lgf1r. Nat Cell Biol. 2012;14(7):659-65.

33. Cai $X$, Cullen BR. The imprinted $H 19$ noncoding RNA is a primary microRNA precursor. RNA-a Publ RNA Soc. 2007:13(3):313-6.

34. Vitak SA, et al. Sequencing thousands of single-cell genomes with combinatorial indexing. Nat Methods. 2017:14(3):302.

35. Garalde DR, et al. Highly parallel direct RNA sequencing on an array of nanopores. Nat Methods. 2018;15(3):201-+.

36. Lee $\mathrm{JH}$, et al. Highly multiplexed subcellular RNA sequencing in situ Science. 2014:343(6177):1360-3.

37. Nazarov PV, et al. RNA sequencing and transcriptome arrays analyses show opposing results for alternative splicing in patient derived samples. BMC Genomics. 2017:18:443.

38. Zhu S, et al. Genome-scale deletion screening of human long non-coding RNAs using a paired-guide RNA CRISPR-Cas9 library. Nat Biotechnol. 2016; 34(12):1279-86.

39. Ho T, et al. Targeting non-coding RNAs with the CRISPR/Cas9 system in human cell lines. Nucleic Acids Res. 2015;43:e173.

40. Li W, et al. Functional roles of enhancer RNAs for oestrogen-dependent transcriptional activation. Nature. 2013;498(7455):516.

41. Davis CP, West JA. Purification of specific chromatin regions using oligonucleotides: capture hybridization analysis of RNA targets (CHART). Methods Mol Biol. 2015;1262:167-82.

42. Engreitz J, Lander ES, Guttman M. RNA antisense purification (RAP) for mapping RNA interactions with chromatin. Methods Mol Biol. 2015;1262: 183-97.

43. Li $X$, et al. GRID-seq reveals the global RNA-chromatin interactome. Nat Biotechnol. 2017;35(10):940-50

44. Gagliardi M, Matarazzo MR. RIP: RNA Immunoprecipitation. Methods Mol Biol. 2016;1480:73-86.

45. Schaukowitch K, Joo JY, Kim TK. UV-RNA immunoprecipitation (UV-RIP) protocol in neurons. Methods Mol Biol. 2017;1468:33-8.

46. Huppertz I, et al. iCLIP: protein-RNA interactions at nucleotide resolution. Methods. 2014:65(3):274-87.

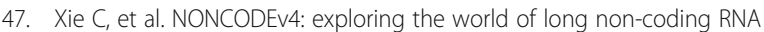
genes. Nucleic Acids Res. 2014;42(D1):D98-D103.

48. Zhou K, et al. ChIPBase v2.0: decoding transcriptional regulatory networks of non-coding RNAs and protein-coding genes from ChIP-seq data. Nucleic Acids Res. 2017;45(D1):D43-50

49. Amaral PP, et al. IncRNAdb: a reference database for long noncoding RNAs. Nucleic Acids Res. 2011;391:D146-51.

50. Volders $P$, et al. LNCipedia: a database for annotated human IncRNA transcript sequences and structures. Nucleic Acids Res. 2013;41(D1):D246-51.

51. Chen $\mathrm{G}$, et al. LnCRNADisease: a database for long-non-coding RNAassociated diseases. Nucleic Acids Res. 2013;41(D1):D983-6. 
52. Li J, et al. Long noncoding RNA MRCCAT1 promotes metastasis of clear cell renal cell carcinoma via inhibiting NPR3 and activating p38-MAPK signaling. Mol Cancer. 2017;16:111.

53. Li Y, et al. Identification of long-non coding RNA UCA1 as an oncogene in renal cell carcinoma. Mol Med Rep. 2016;13(4):3326-34.

54. Xiong J, et al. High expression of long non-coding RNA IncRNA-ATB is correlated with metastases and promotes cell migration and invasion in renal cell carcinoma. Jpn J Clin Oncol. 2016;46(4):378-84.

55. Wang $L$, et al. Down-regulated long non-coding RNA H19 inhibits carcinogenesis of renal cell carcinoma. Neoplasma. 2015;62(3):412-8.

56. He X, et al. Knockdown of Long noncoding RNA FTX inhibits proliferation, migration, and invasion in renal cell carcinoma cells. Oncol Res. 2017;25(2): 157-66.

57. Zhai W, et al. Differential regulation of LnCRNA-SARCC suppresses VHLmutant RCC cell proliferation yet promotes VHL-normal RCC cell proliferation via modulating androgen receptor/HIF-2 alpha/C-MYC axis under hypoxia. Oncogene. 2016;35(37):4866-80.

58. Xue $\mathrm{S}$, et al. Decreased expression of long non-coding RNA NBAT-1 is associated with poor prognosis in patients with clear cell renal cell carcinoma. Int J Clin Exp Pathol. 2015;8(4):3765-74.

59. Cao $Y$, et al. Downregulation of IncRNA CASC2 by microRNA-21 increases the proliferation and migration of renal cell carcinoma cells. Mol Med Rep. 2016:14(1):1019-25.

60. Blondeau JJC, et al. Identification of novel long non-coding RNAs in clear cell renal cell carcinoma. Clin Epigenetics. 2015;7:10.

61. Fachel AA, et al. Expression analysis and in silico characterization of intronic long noncoding RNAs in renal cell carcinoma: emerging functional associations. Mol Cancer. 2013;12:140.

62. Qu L, et al. Exosome-transmitted IncARSR promotes Sunitinib resistance in renal cancer by acting as a competing endogenous RNA. Cancer Cell. 2016; 29(5):653-68.

63. Geng $X$, et al. The effect of long noncoding RNA GAS5 on apoptosis in renal ischemia/reperfusion injury. Nephrology (Carlton). 2018. https://doi. org/10.1111/nep.13476.

64. Lelli $A$, et al. Induction of long noncoding RNA MALAT1 in hypoxic mice. Hypoxia. 2015;3:45-52

65. Koelling $M$, et al. Hypoxia-induced long non-coding RNA Malat1 is dispensable for renal ischemia/reperfusion-injury. Sci Rep. 2018;8:3438.

66. Yu T, et al. RANTES mediates kidney ischemia reperfusion injury through a possible role of HIF-1 alpha and LncRNA PRINS. Sci Rep. 2016;6:18424.

67. Lorenzen JM, et al. Circulating long noncoding RNA TapSAKI is a predictor of mortality in critically ill patients with acute kidney injury. Clin Chem. 2015;61(1):191-201.

68. Feng $M$, et al. TGF-beta mediates renal fibrosis via the Smad3-Erbb4-IR Long noncoding RNA Axis. Mol Ther. 2018;26(1):148-61.

69. Wang J, et al. IncRNA ZEB1-AS1 was suppressed by p53 for renal fibrosis in diabetic nephropathy. Mol Therapy-Nucleic Acids. 2018;12:741-50.

70. Xie $\mathrm{H}$, et al. Long non-coding RNA-H19 antagonism protects against renal fibrosis. Oncotarget. 2016;7(32):51473-81.

71. Chen W, et al. Effects of Long non-coding RNA LINC00963 on renal interstitial fibrosis and oxidative stress of rats with chronic renal failure via the Foxo signaling pathway. Cell Physiol Biochem. 2018;46(2):815-28.

72. Zhou $\mathrm{H}$, et al. LnCRNA HOTAIR promotes renal interstitial fibrosis by regulating Notch1 pathway via the modulation of miR-124. Nephrology (Carlton, Vic). 2018

73. Chen S, et al. Microarray analysis of long noncoding RNA expression patterns in diabetic nephropathy. J Diabetes Complicat. 2017;31(3):569-76.

74. Wang $M$, et al. A novel long non-coding RNA CYP4B1-PS1-001 regulates proliferation and fibrosis in diabetic nephropathy. Mol Cell Endocrinol. 2016; 426(C):136-45

75. Wang M, et al. Long non-coding RNA ENSMUST00000147869 protects mesangial cells from proliferation and fibrosis induced by diabetic nephropathy. Endocrine. 2016:54(1):81-92.

76. Gao J, et al. LncRNA-NR_033515 promotes proliferation, fibrogenesis and epithelial-to-mesenchymal transition by targeting miR-743b-5p in diabetic nephropathy. Biomed Pharmacother. 2018;106:543-52.

77. Cheng J, et al. Inhibition of IncRNA DIx6os1 decreases cell proliferation and fibrosis and increases cell apoptosis in diabetic nephropathy. Int J Clin Exp Pathol. 2018;11(7):3302-9.
78. Li Z, et al. LnCRNA LINC00968 accelerates the proliferation and fibrosis of diabetic nephropathy by epigenetically repressing p21 via recruiting EZH2. Biochem Biophys Res Commun. 2018;504(2):499-504.

79. Wu D, et al. Downregulation of IncRNA MALAT1 contributes to renal functional improvement after duodenal-jejunal bypass in a diabetic rat model. J Physiol Biochem. 2018;74(3):431-9.

80. Lei $X$, et al. Astragaloside IV/IncRNA-TUGI/TRAF5 signaling pathway participates in podocyte apoptosis of diabetic nephropathy rats. Drug Design Dev Ther. 2018;12:2785-93.

81. Duan $L$, et al. Long noncoding RNA TUG1 alleviates extracellular matrix accumulation via mediating microRNA-377 targeting of PPAR gamma in diabetic nephropathy. Biochem Biophys Res Commun. 2017:484(3):598-604.

82. Long J, et al. Long noncoding RNA Tug1 regulates mitochondrial bioenergetics in diabetic nephropathy. J Clin Investig. 2016;126(11):4205-18.

83. Sui $W$, et al. Altered long non-coding RNA expression profile in patients with IgA-negative mesangial proliferative glomerulonephritis. Int J Mol Med. 2012;30(1):173-8.

84. Aboudehen K, et al. Long noncoding RNA Hoxb3os is dysregulated in autosomal dominant polycystic kidney disease and regulates mTOR signaling. J Biol Chem. 2018;293(24):9388-98.

85. Zhang $F$, et al. Identification of the long noncoding RNA NEAT1 as a novel inflammatory regulator acting through MAPK pathway in human lupus. J Autoimmun. 2016;75:96-104

86. Chen W, et al. Microarray analysis of long non-coding RNA expression in human acute rejection biopsy samples following renal transplantation. Mol Med Rep. 2014;10(4):2210-6.

87. Lorenzen $\mathrm{JM}$, et al. Long noncoding RNAs in urine are detectable and may enable early detection of acute $T$ cell-mediated rejection of renal allografts. Clin Chem. 2015;61(12):1505-14.

88. Ge Y, et al. A molecular signature of two Long non-coding RNAs in peripheral blood predicts acute renal allograft rejection. Cell Physiol Biochem. 2017:44(3):1213-23.

89. Shang $D$, et al. Profiling of mRNA and long non-coding RNA of urothelial cancer in recipients after renal transplantation. Tumor Biol. 2016;37(9): 12673-84.

90. Qiu J, et al. Transforming growth factor- activated long non-coding RNA ATB plays an important role in acute rejection of renal allografts and may impacts the postoperative pharmaceutical immunosuppression therapy. Nephrology. 2017;22(10):796-803.

91. Zou X, et al. PRINS long noncoding RNA involved in IP-10-mediated allograft rejection in rat kidney transplant. Transplant Proc. 2018:50(5):1558-65.

92. Arun $\mathrm{G}$, et al. Differentiation of mammary tumors and reduction in metastasis upon Malat1 IncRNA loss. Genes Dev. 2016;30(1):34-51.

Ready to submit your research? Choose BMC and benefit from:

- fast, convenient online submission

- thorough peer review by experienced researchers in your field

- rapid publication on acceptance

- support for research data, including large and complex data types

- gold Open Access which fosters wider collaboration and increased citations

- maximum visibility for your research: over $100 \mathrm{M}$ website views per year

At $\mathrm{BMC}$, research is always in progress.

Learn more biomedcentral.com/submissions 